\title{
Recombinant Factor VIla Analog NN1731 (V158D/E296V/M298Q- FVIla) Enhances Fibrin Formation, Structure and Stability in Lipidated Hemophilic Plasma
}

\author{
Laura D. Gray ${ }^{1}$, Michael A. Hussey ${ }^{2}$, Brittany M. Larson ${ }^{1}$, Kellie R. Machlus ${ }^{1}$, Robert A. \\ Campbell $^{1}$, Gary Koch ${ }^{2}$, Mirella Ezban ${ }^{3}$, Ulla Hedner ${ }^{3}$, and Alisa S. Wolberg ${ }^{1}$ \\ ${ }^{1}$ Department of Pathology and Laboratory Medicine, University of North Carolina at Chapel Hill, \\ Chapel Hill, NC, USA \\ ${ }^{2}$ Department of Biostatistics, University of North Carolina at Chapel Hill, Chapel Hill, NC, USA \\ ${ }^{3}$ Novo Nordisk A/S, Novo Nordisk Park, Maaloev, Denmark
}

\section{SUMMARY}

Introduction-The bypassing agent recombinant factor VIIa (rFVIIa) is efficacious in treating bleeding in hemophilia patients with inhibitors. Efforts have focused on the rational engineering of rFVIIa variants with increased hemostatic potential. One rFVIIa analog (V158D/E296V/M298QFVIIa, NN1731) improves thrombin generation and clotting in purified systems, whole blood from hemophilic patients and factor VIII-deficient mice.

Methods-We used calibrated automated thrombography and plasma clotting assays to compare effects of bypassing agents (rFVIIa, NN1731) on hemophilic clot formation, structure, and ability to resist fibrinolysis.

Results-Both rFVIIa and NN1731 shortened the clotting onset and increased the maximum rate of fibrin formation and fibrin network density in hemophilic plasma clots. In the presence of tissue plasminogen activator, both rFVIIa and NN1731 shortened the time to peak turbidity (TTPeak ${ }_{t_{P A}}$ ) and increased the area under the clot formation curve $\left(\mathrm{AUC}_{\mathrm{tPA}}\right)$. Phospholipids increased both rFVIIa and NN1731 activity in a lipid concentration-dependent manner. Estimated geometric mean concentrations of rFVIIa and NN1731 producing similar onset, rate, TTPeak ${ }_{\mathrm{tPA}}$, and $\mathrm{AUC}_{\mathrm{tPA}}$ as seen with $100 \%$ factors VIII and IX were: $24.5,74.3,29.7$, and $37.1 \mathrm{nM} \mathrm{rFVIIa}$, and 8.6, 31.2, 9.0, and $11.3 \mathrm{nM} \mathrm{NN} 1731$, respectively. In each case, the NN1731 concentration was significantly lower than rFVIIa.

Conclusions-These findings suggest that like rFVIIa, NN1731 improves the formation, structure, and stability of hemophilic clots. Higher lipid concentrations may facilitate assessment of both rFVIIa and NN1731 activity. NN1731 appears likely to support rapid clot formation in tissues with high endogenous fibrinolytic activity.

(C) 2011 Elsevier Ltd. All rights reserved.

Address correspondence to: Alisa S. Wolberg, Ph. D., Department of Pathology and Laboratory Medicine, University of North Carolina at Chapel Hill, 815 Brinkhous-Bullitt Building, CB \#7525, Chapel Hill, NC 27599-7525, phone: (919) 966-8430, fax: (919) 966-6718, alisa_wolberg@med.unc.edu.

Publisher's Disclaimer: This is a PDF file of an unedited manuscript that has been accepted for publication. As a service to our customers we are providing this early version of the manuscript. The manuscript will undergo copyediting, typesetting, and review of the resulting proof before it is published in its final citable form. Please note that during the production process errors may be discovered which could affect the content, and all legal disclaimers that apply to the journal pertain. 


\section{Keywords}

hemophilia; recombinant factor VIIa; fibrinogen; fibrinolysis; NN1731

\section{INTRODUCTION}

Recombinant factor VIIa (rFVIIa, NovoSeven; Novo Nordisk A/S, Copenhagen, Denmark) is effective in treating bleeding in hemophilic patients with inhibitors.[1] At pharmacologic doses (90-270 $\mu \mathrm{g} / \mathrm{kg}$, or plasma levels of $25-75 \mathrm{nM}$ ), rFVIIa binds to activated platelets and increases thrombin generation on the platelet surface in a tissue factor (TF)-independent process.[2,3] Using in vitro assays with reconstituted model systems, hemophilic plasma, and whole blood, we and others have shown that rFVIIa shortens the lag time and increases the rate of thrombin generation $[2,4,5]$. Recombinant FVIIa also shortens the onset time of fibrin formation and normalizes the structure and porosity of fibrin networks formed under hemophilic conditions.[4, 6] Recombinant FVIIa improves fibrin formation in the presence of tissue plasminogen activator (tPA) and plasmin[4, 5], suggesting it may improve formation of the primary clot as well as subsequent clots if the primary clot is prematurely lysed.

Recombinant FVIIa treatment with standard doses is effective in greater than $90 \%$ of patients; however, clinical experience with rFVIIa suggests dosing must be individualized to achieve optimal treatment outcomes in a subset of patients.[7, 8] Recombinant FVIIa analogs with increased activity may promote greater thrombin generation and clot stability than rFVIIa, and support hemostasis in these patients. Several rFVIIa analogs have been generated that express substantially higher TF-independent activity than rFVIIa in in vitro assays. [5, 9, 10] One of these rFVIIa analogs, NN1731, increases the thrombin generation rate, shortens the clotting time, and increases clot stability in a purified in vitro model of hemophilia at up to 50-fold lower concentrations than are required of rFVIIa.[5] NN1731 also dose-dependently reduces blood loss in murine tail bleeding models of hemophilia significantly faster and at lower doses than are required of rFVIIa.[11, 12] Based on these findings, NN1731 was recently tested in a phase I dose escalation trial in healthy males and found to be safe and well-tolerated at doses up to $30 \mu \mathrm{g} / \mathrm{kg}(\sim 8.4 \mathrm{nM})$.[13] A phase II trial is now complete with good efficacy and no safety concerns observed with doses of NN1731 tested up to $80 \mu \mathrm{g} / \mathrm{kg}$.[14] Given the diversity of assays to measure rFVIIa activity, it is of interest to identify effective in vitro methods for assessing rFVIIa and NN1731 activity. It is also of significant interest to identify concentrations of NN1731 that produce similar or improved effects as rFVIIa, and determine whether a given concentration of either bypassing agent similarly "corrects" all parameters of clotting (formation, structure, and stability).

In the current study we compared the effects of rFVIIa and NN1731 on the formation, structure and fibrinolytic stability of hemophilic plasma clots. Results were compared to $100 \%$ levels of factors VIII and IX. Phospholipids increased the ability of both rFVIIa and NN1731 to modulate hemophilic plasma clots in a concentration-dependent manner. Both bypassing agents improved clotting, fibrin structure, and fibrinolytic resistance of lipidated hemophilic plasma. Concentrations of rFVIIa and NN1731 were identified that produced similar effects as $100 \%$ factor levels; in each case NN1731 exhibited significantly higher activity than rFVIIa. 


\section{MATERIALS AND METHODS}

\section{Proteins and reagents}

Factor IX was purified, treated with an inhibitor mixture, isolated on Q Sepharose with $\mathrm{CaCl}_{2}$ elution and dialyzed, as described.[4] Factor VIII (Hemofil M, Baxter) was generously provided by Dr. Dougald M. Monroe (University of North Carolina). TPA was from American Diagnostica (Stamford, CT, USA). RFVIIa and NN1731 (rFVIIa V158D/ E296V/M298Q) were from Novo Nordisk and were fully carboxylated at the first 9 out of 10 potential Gla positions and partially carboxylated at the $10^{\text {th }}$ potential Gla position (amino acid 35). NN1731 contains aspartate substituted for valine, valine for glutamate, and glutamine for methionine at positions 158, 296, and 298, respectively.[9] Hemophilic platelet-poor plasmas (PPP) from nine individuals (eight hemophilia A, one hemophilia B) with $<1 \%$ factor were purchased from HRF (Raleigh, NC, USA). TF for calibrated automated thrombography (PRP reagent) was from Diagnostica Stago (Parsippany, NJ). Monocytes were isolated and treated with lipopolysaccharide overnight (Serotype 0128:B12, Sigma, St. Louis, MO, USA) to induce TF expression for clotting assays.[4] AlexaFluorconjugated fibrinogen was prepared as described.[15]

\section{Phospholipid vesicles}

Phosphatidylcholine, phosphatidylethanolamine, and phosphatidylserine were from Avanti Polar Lipids (Alabaster, AL, USA). Large unilamellar vesicles (41\% phosphatidylcholine / 44\% phosphatidylethanolamine $/ 15 \%$ phosphatidylserine) were made as described.[16] Briefly, lipids were combined, dried under nitrogen gas, and resuspended in cyclohexane. Resuspended lipids were lyophilized, resuspended in $20 \mathrm{mM} \mathrm{N}$-2-hydroxyethylpiperazineN'-2-ethanesulfonic acid (HEPES) pH 7.4, $150 \mathrm{mM} \mathrm{NaCl}$ containing $1 \mathrm{mM}$ ethylenediamine tetraacetic acid, and extruded through a $0.2 \mu \mathrm{m}$ filter ten times.

\section{Thrombin generation}

Thrombin generation was monitored in duplicate or triplicate in each experiment by calibrated automated thrombography.[17] Briefly, TF (PRP reagent, $1 \mathrm{pM}$, final) and phospholipids (at concentrations indicated) were added to plasmas and thrombin generation was initiated by automatically dispensing fluorogenic substrate (Z-Gly-Gly-Arg-AMC) in $\mathrm{CaCl}_{2}(416 \mu \mathrm{M}$ and $16 \mathrm{mM}$, final, respectively). Thrombin was calibrated against wells containing $\alpha 2$-macroglobulin/thrombin complex and plasma. Thrombin parameters were calculated using Thrombinoscope software version 3.0.0.29 (Thrombinoscope BV, Maastricht, Netherlands).

\section{Characterization of fibrin formation and lysis}

Clotting was initiated by incubating recalcified ( $20 \mathrm{mM}$, final) PPP with TF-bearing monocytes (providing $\sim 1 \mathrm{pM} \mathrm{TF}[18]$ ) and phospholipids (at concentrations indicated) in the presence or absence of tPA ( $1 \mu \mathrm{g} / \mathrm{mL}$, final) at room temperature. RFVIIa and NN1731 were added as indicated. Final volumes were $100 \mu \mathrm{L}$. Clot formation and lysis were detected by turbidity at $405 \mathrm{~nm}$ with a SpectraMax 340Plus plate reader (Molecular Devices, Sunnyvale, CA, USA). The clot formation onset was the time to the inflection point prior to the turbidity increase. The maximum slope was the slope of a line fitted to the maximum rate of turbidity increase ("Vmax") using 5-10 points to determine the line. For lysis experiments, the time to peak turbidity (TTPeak $\mathrm{tPA}_{\mathrm{TA}}$ ) was the time to the inflection point at peak turbidity, and the area under the curve $\left(\mathrm{AUC}_{\mathrm{tPA}}\right)$ was the sum of trapezoids formed by turbidity curves less a baseline established by the lowest measurement taken (Kaleidagraph 4.1.0, Synergy

Software, http://www.synergy.com). 


\section{Fibrin structure analysis}

Clots were formed as above in Labtek II glass chamber slides and scanned with a Zeiss LSM5 Pascal confocal laser scanning microscope (Carl Zeiss, Inc) linked to a Zeiss inverted microscope equipped with a Zeiss $63 \times$ oil immersion plan apo-chromatic lens, as described. [15] Thirty optical sections (1024×1024 pixels) were collected at $0.36-\mu \mathrm{m}$ intervals in the $\mathrm{z}$ axis, beginning just above the cell surface. Images were processed using 3D deconvolution algorithms in AutoQuant's Autodeblur software (Media Cybernetics Inc, Bethesda, MD, USA) prior to image analysis.[15] Fibrin density (area covered by fibrin) was determined using Image $\mathrm{J} 1.39 \mathrm{t}$ by summing individual sections to create $\mathrm{z}$ projections, thresholding, and calculating the area.

\section{Statistical analysis}

Descriptive statistics for clotting onset, fibrin formation rate, fibrin density, TTPeak ${ }_{\mathrm{tPA}}$, and $\mathrm{AUC}_{\mathrm{tPA}}$ were summarized using graphical illustrations for mean \pm standard error of the mean (SEM) response at each concentration for rFVIIa and NN1731. Thrombin generation peaks were analyzed by two-way analysis of variance (ANOVA) using subject and condition as factors. A Dunnett's post hoc test was then used to categorically identify phospholipid concentrations that produced significantly different peaks versus no phospholipids. For fibrin formation, network density, and lysis assays, analysis of covariance (ANCOVA) models that controlled for hemophilia were applied to estimate the response curves for log-transformed assay measures of rFVIIa and NN1731 relative to $100 \%$ factors VIII and IX. The results from these models were used to estimate the geometric mean concentrations of rFVIIa and NN1731 required to produce similar readouts as $100 \%$ factors VIII and IX under the assumption of parallel linear assay relationships (on a logscale). Under the parallel assay assumption, rFVIIa is viewed as a dilution of a more potent analog, i.e. NN1731. Relative activity of the analog versus rFVIIa was then estimated assuming a common response rate. The $95 \%$ confidence intervals were computed using Fieller's method.[19, 20] A detailed Appendix describing the statistical method is available online. A p-value $<0.05$ was considered significant while a $95 \%$ confidence interval that excludes one (zero on log-scale) was considered evidence that the analog was significantly more active.

\section{RESULTS}

\section{Effect of phospholipids on thrombin generation}

We first titrated phospholipids $(0-300 \mu \mathrm{M})$ into hemophilia A or B PPP, or hemophilia A or B PPP supplemented with $50 \mathrm{nM}$ rFVIIa or $5 \mathrm{nM}$ NN1731. Fifty nM rFVIIa corresponds to plasma levels of rFVIIa achieved following standard infusion of $180 \mu \mathrm{g} / \mathrm{kg}$. We used $100 \%$ factor VIII or IX (1 U/mL or $70 \mathrm{nM}$, respectively) to model "normal" (positive control) in our experiments. Thrombin generation was initiated and measured by calibrated automated thrombography, as described in Methods. We observed a slightly prolonged lagtime in the absence of exogenous lipid, but otherwise little effect of phospholipid on the thrombin lagtime under any condition (data not shown). In contrast, thrombin peak showed a significant $(\mathrm{p}<0.0001)$ dependence on phospholipid concentration; compared to no added phospholipid, 80 and $2 \mu \mathrm{M}$ phospholipid significantly increased the thrombin peak in hemophilic plasma (Figure 1A) and in the presence of 100\% factors VIII and IX (Figure 1B), respectively. Both rFVIIa and NN1731-mediated thrombin generation in hemophilic PPP also showed significant $(\mathrm{p}<0.0001)$ dependence on phospholipid concentration (Figures $1 \mathrm{C}, 1 \mathrm{D}$ ); peaks from reactions containing $\geq 40 \mu \mathrm{M}$ phospholipid were significantly higher than no phospholipids for both molecules. These findings are consistent with studies of rFVIIa-dependent thrombin generation[21, 22], but extend these observations to NN1731. 


\section{Effect of phospholipids on clot formation}

Given the dependence of both rFVIIa- and NN1731-dependent thrombin generation on phospholipid concentration, we then determined the effects of lipid concentration on fibrin formation. We titrated phospholipid vesicles $(4-300 \mu \mathrm{M})$ into hemophilia A or B PPP, or hemophilia A or B PPP supplemented with 100\% factors VIII or IX, respectively, $50 \mathrm{nM}$ rFVIIa, or $5 \mathrm{nM}$ NN1731, and monitored clotting by turbidity, as described in Methods. Hemophilic samples clotted with prolonged onset and reduced maximum slope (clot formation rate), compared to $100 \%$ factors VIII and IX (Figures 2A, 2B).[4, 5] The onset and rate of fibrin formation in the presence or absence of factors VIII or IX were only minimally affected by phospholipid concentrations between 4-300 $\mu \mathrm{M}$, whereas the onset and rate of rFVIIa- and NN1731-dependent fibrin formation were progressively shortened and increased, respectively, by additional phospholipid. Discord between the thrombin generation lagtime and clot formation onset is thought to reflect differences in the sensitivity of assay parameters to extrinsic and intrinsic activity, as well as differences in using thrombin chromogenic or fluorogenic activity versus thrombin conversion of fibrinogen to fibrin as an assay readout.[23] In particular, fibrinogen conversion reflects a thrombin concentration threshold phenomenon; lower concentrations of thrombin can convert fibrinogen to fibrin than can be detected by thrombin substrates. Because $125 \mu \mathrm{M}$ phospholipid was the lowest phospholipid concentration that produced a similar fibrin polymerization rate in rFVIIa-treated samples as $100 \%$ factors VIII and IX, this phospholipid concentration was used in subsequent clotting studies.

\section{Effect of rFVIla and NN1731 on clotting}

We next followed fibrin formation in hemophilic PPP supplemented with $125 \mu \mathrm{M}$ phospholipids and rFVIIa (1-300 nM) or NN1731 (0.5-25 nM) (Figure 3). Addition of either rFVIIa or NN1731 to lipidated hemophilic plasma significantly $(\mathrm{p}<0.05)$ shortened the onset (Figure 3B) and increased the rate (Figure 3C) of fibrin formation in a concentration-dependent manner (Table I). From these data we determined the estimated geometric mean concentrations of rFVIIa and NN1731 that produced similar clotting onset and rate as 100\% factors VIII/IX (Table I), and used these data to calculate the relative potency of NN1731 compared to rFVIIa (Table II).

\section{Effect of rFVIla and NN1731 on fibrin structure}

Growing evidence suggests fibrin structure is a sensitive reflection of thrombin levels present during fibrin formation and an integral determinant of the clot's mechanical and fibrinolytic stability.[24] Clots formed from hemophilic plasma and whole blood exhibit an abnormally coarse, porous network of thick fibers compared to normal, and rFVIIa increases fibrin network density.[4, 6] A recent preliminary report suggested NN1731 can similarly decrease fibrin network porosity of hemophilic clots.[25] Herein we tested a broader range of rFVIIa and NN1731 concentrations to compare the dose-dependency of rFVIIa and NN1731 on network density (Figure 4A). Our data (Table I, Figure 4B) are consistent with the prior report, but suggest considerable variability with density measurements; the wide confidence intervals reflect inter-individual differences in plasma activity, variability in network structure, and fewer replicates due to the time consuming nature of these assessments. Consistent with previous findings[4], fibers present in hemophilic clots appeared thicker than those formed in the presence of $100 \%$ factors VIII and IX. However, because fiber diameter $(200-400 \mathrm{~nm})$ is at the lower resolution limit of LSCM[26, 27], we did not specifically quantify fibrin diameter in these clots. 


\section{Effect of rFVIla and NN1731 on fibrinolysis}

It has been proposed that delayed bleeding in hemophilic patients results, at least in part, from increased susceptibility of hemophilic clots to premature fibrinolysis.[28] We examined the ability of rFVIIa and NN1731 to improve fibrin formation and stability in the presence of tPA. As shown in Figure 5, clot formation (turbidity increase) and subsequent lysis (turbidity decrease) are observed in the presence of 100\% factors VIII and IX levels; however, hemophilic conditions are not sufficiently procoagulant to produce fibrin. Both rFVIIa and NN1731 significantly $(\mathrm{p}<0.05)$ shortened the TTPeak $\mathrm{t}_{\mathrm{tPA}}$ (peak incorporation of fibrin into the clot, Figure 5B) and $\mathrm{AUC}_{\mathrm{tPA}}$ (clot stability, Figure 5C); however, the estimated geometric mean concentrations of NN1731 required were lower than those of rFVIIa (Table I). These findings are consistent with increased procoagulant activity of NN1731 relative to rFVIIa (Table II).

\section{DISCUSSION}

Since the introduction of rFVIIa as a bypassing agent for treating bleeds in hemophilic patients with inhibitors, considerable effort has focused on developing improved variants of this molecule. The need for these improvements stems, in part, from observations that rFVIIa dosing must be individualized in certain patients to achieve optimal treatment outcomes. $[7,8]$ Molecules currently in basic and clinical development include a factor VIIaalbumin fusion protein ("rVIIa-FP")[29], factor VIIa formulated with PEGylated liposomes[30], GlycoPEGylated rFVIIa[31], and recombinant analogs of [rFVIIa-QE[32] rFVIIa(VEAY)[33], and NN1731[5, 9, 11, 12, 34-37]]. NN1731, in particular, has been evaluated in purified systems[36], cell-based reconstituted models of hemophilia[5], hemophilic whole blood[34, 35], and in vivo studies in hemophilic mice[11, 12] and dogs[37, 38]. These studies have suggested NN1731 induces hemostasis in hemophilic conditions, but with a faster onset and at lower concentrations than are required of rFVIIa. Our current study using lipidated plasma from individual hemophilic patients supports and extends these observations on the mechanism of action of rFVIIa and NN1731. We identified rFVIIa and NN1731 estimated geometric mean concentrations that produced similar effects as $100 \%$ factor VIII and IX on the onset and formation rate of hemophilic clots (Figure 3), fibrin network structure (Figure 4), and resistance to fibrinolysis (Figure 5). In each of these assays, NN1731 demonstrated at least 2.4-fold higher fibrin-promoting activity than rFVIIa (Table II).

Dosing in the initial in vivo rFVIIa studies was based on theoretical calculations of the rFVIIa concentration needed to saturate TF at injury sites; however, studies showing that rFVIIa functions via binding to the platelet surface[2] provide a rationale for the supraphysiologic doses necessary to achieve efficacy in patients. [39] It has been notoriously difficult to identify an in vitro assay in which rFVIIa has similar effects as $100 \%$ factor VIII and IX levels. While thrombin generation assays are sensitive to rFVIIa activity, studies evaluating thrombin generation have not shown normalization by rFVIIa. [4, 5, 34, 40, 41] It is therefore of considerable interest that our clotting assays demonstrated several parameters in which rFVIIa produces similar effects as $100 \%$ factor VIII and IX levels. Specifically, under the conditions of our assays, the estimated geometric mean rFVIIa concentrations producing similar readouts as $100 \%$ factors VIII and IX for onset and rate in the clot formation assays, and TTPeak $\mathrm{tPA}_{\mathrm{tPA}}$ and $\mathrm{AUC}_{\mathrm{tPA}}$ in the fibrinolysis assays were 24.5, 74.3, 29.7, and $37.1 \mathrm{nM}$, respectively (Table I). Additional repeated measures ANCOVA models were explored to account for potential dependence of measures of clot formation and fibrinolysis within an assay, but these models did not produce appreciably different estimates from those in Tables I and II. Interestingly, for both models, these concentrations are close to the expected plasma concentrations achieved following a dose of $90 \mu \mathrm{g} / \mathrm{kg}$ rFVIIa $(\sim 25 \mathrm{nM})$, which is efficacious in $70-90 \%$ of patients.[42] These data suggest the 
conditions currently used for most thrombin generation tests might be further optimized to provide the best readout for identifying clinically-effective bypassing agent concentrations. Further studies are warranted to determine whether this lipidated plasma-based clotting assay can be used to predict dosing to induce hemostasis in hemophilic patients.

Although it is probably ideal to measure rFVIIa activity in the presence of platelets, logistical difficulties in collecting and handling platelet-rich plasma have prevented the wide-spread adoption of this approach in laboratory assays. Synthetic phospholipid vesicles can be substituted for platelets in thrombin generation assays, and recent studies have shown that in the absence of platelets, exogenous phospholipids are essential for rFVIIa-mediated thrombin generation.[21, 22, 43] We extended these findings to show NN1731 also produces shorter onsets and increased clot formation rates with increasing phospholipids (Figure 2). Thus, although platelets are the physiologic site of rFVIIa activity in hemophilia, phospholipid vesicles with similar phosphatidylserine content as platelets[44] such as those used in this study, may be a practical and effective substitute in preclinical dosing studies to screen other novel factor VIIa variants. Since we and others[21, 22] have shown both thrombin generation and clot formation are phospholipid concentration-dependent, the lipid concentrations used in these assays should be optimized for these assessments. Of course, since the response to rFVIIa has been proposed to stem, in part, from the quality of the endogenous platelet population in patients[1,45], a "normalizing" concentration of rFVIIa or NN1731 in assays utilizing lipidated PPP may not reflect doses needed to achieve efficacy in "non-responder" patients. In these cases, performing in vitro clotting assays with platelet-rich plasma may provide more tailored information on dosing than assays using phospholipids.

In conclusion, our data show the significant dependence of thrombin generation and fibrin formation on phospholipid concentration, and suggest that optimizing the lipid concentration may provide clinically-relevant information about bypass factor dosing. Both rFVIIa and NN1731 significantly improve fibrin formation, structure and stability in hemophilic clots; however, effects of NN1731 manifest at significantly lower concentrations than are required of rFVIIa. Finally, our findings suggest NN1731 will have clinical utility as a hemostatic agent in hemophilic inhibitor patients refractory to standard rFVIIa concentrations. These data support continued evaluation of this agent in in vitro and in vivo studies.

\section{Supplementary Material}

Refer to Web version on PubMed Central for supplementary material.

\section{Acknowledgments}

The authors thank Drs. Maureane Hoffman, Dougald M. Monroe, and Yesim Dargaud for their thoughtful contributions.

Grant support: Novo Nordisk (ASW), National Hemophilia Foundation (ASW), and National Institutes of Health (R01HL094740, ASW).

\section{REFERENCES}

1. Hedner U. Recombinant factor VIIa: its background, development and clinical use. Curr Opin Hematol. 2007; 14:225-229. [PubMed: 17414211]

2. Monroe DM, Hoffman M, Oliver JA, Roberts HR. Platelet activity of high-dose factor VIIa is independent of tissue factor. Brit J Haematol. 1997; 99:542-547. [PubMed: 9401063]

3. Monroe DM, Hoffman M, Oliver JA, Roberts HR. A possible mechanism of action of activated factor VII independent of tissue factor. Blood Coag Fibrinol. 1998; 9:S15-S20. 
4. Wolberg AS, Allen GA, Monroe DM, Hedner U, Roberts HR, Hoffman M. High dose factor VIIa enhances clot stability in a model of hemophilia B. Brit J Haematol. 2005; 131:645-655. [PubMed: 16351642]

5. Allen GA, Persson E, Campbell RA, Ezban M, Hedner U, Wolberg AS. A variant of recombinant factor VIIa with enhanced procoagulant and antifibrinolytic activities in an in vitro model of hemophilia. Arterioscler Thromb Vasc Biol. 2007; 27:683-689. [PubMed: 17204663]

6. He S, Blomback M, Jacobsson Ekman G, Hedner U. The role of recombinant factor VIIa (FVIIa) in fibrin structure in the absence of FVIII/FIX. J Thromb Haemost. 2003; 1:1215-1219. [PubMed: 12871322]

7. Kenet G, Lubetsky A, Luboshitz J, Martinowitz U. A new approach to treatment of bleeding episodes in young hemophilia patients: a single bolus megadose of recombinant activated factor VII (NovoSeven). J Thromb Haemost. 2003; 1:450-455. [PubMed: 12871449]

8. Cooper HA, Jones CP, Campion E, Roberts HR, Hedner U. Rationale for the use of high dose rFVIIa in a high-titre inhibitor patient with haemophilia B during major orthopaedic procedures. Haemophilia. 2001; 7:517-522. [PubMed: 11554944]

9. Persson E, Kjalke M, Olsen OH. Rational design of coagulation factor VIIa variants with substantially increased intrinsic activity. PNAS USA. 2001; 98:13583-13588. [PubMed: 11698657]

10. Lisman T, de Groot PG, Lambert T, Rojkjaer R, Persson E. Enhanced in vitro procoagulant and antifibrinolytic potential of superactive variants of recombinant factor VIIa in severe hemophilia A. J Thromb Haemost. 2003; 1:2175-2178. [PubMed: 14521601]

11. Tranholm M, Kristensen K, Kristensen AT, Pyke C, Rojkjaer R, Persson E. Improved hemostasis with superactive analogs of factor VIIa in a mouse model of hemophilia A. Blood. 2003; 102:3615-3620. [PubMed: 12869500]

12. Holmberg HL, Lauritzen B, Tranholm M, Ezban M. Faster onset of effect and greater efficacy of NN1731 compared with rFVIIa, aPCC and FVIII in tail bleeding in hemophilic mice. J Thromb Haemost. 2009; 7:1517-1522. [PubMed: 19566792]

13. Moss J, Scharling B, Ezban M, Moller Sorensen T. Evaluation of the safety and pharmacokinetics of a fast-acting recombinant FVIIa analogue, NN1731, in healthy male subjects. J Thromb Haemost. 2009; 7:299-305. [PubMed: 19138379]

14. de Paula E, Kavakli K, Mahlangu J, Ayob Y, Lentz SR, Morfini M, et al. Safety and preliminary efficacy of recombinant activated FVII analog (NN1731) In the treatment of joint bleeds In congenital hemophilia patients with inhibitors. Blood. 2010; 116:317. [PubMed: 20308595]

15. Campbell RA, Overmyer KA, Bagnell CR, Wolberg AS. Cellular procoagulant activities dictate clot structure and stability as a function of distance from the cell surface. Arterio Thromb Vasc Biol. 2008; 28:2247-2254.

16. Hope MJ, Bally MB, Webb G, Cullis PR. Production of large unilamellar vesicles by a rapid extrusion procedure. Characterization of size distribution, trapped volume and ability to maintain a membrane potential. Biochem Biophys Acta. 1985; 812:55-65.

17. Machlus KR, Colby EA, Wu JR, Koch GG, Key NS, Wolberg AS. Effect of tissue factor, thrombomodulin, and elevated clotting factor levels on thrombin generation in the calibrated automated thrombogram. Thromb Haemost. 2009; 102:936-944. [PubMed: 19888532]

18. Oliver JA, Monroe DM, Church FC, Roberts HR, Hoffman M. Activated protein C cleaves factor Va more efficiently on endothelium than on platelet surfaces. Blood. 2002; 100:539-546. [PubMed: 12091346]

19. Zerbe GO. On Fieller's theorem and the general linear model. The American Statistician. 1978; 32

20. Stokes, ME.; Davis, CS.; Koch, GG. 2nd ed. Cary: SAS Publishing and John Wiley \& Sons, Inc; 2000. Categorical data analysis using the SAS system.

21. Butenas S, Brummel KE, Paradis SG, Mann KG. Influence of factor VIIa and phospholipids on coagulation in "acquired" hemophilia. [comment]. Arterioscler Thromb Vasc Biol. 2003; 23:123129. [PubMed: 12524235]

22. Livnat T, Zivelin A, Martinowitz U, Salomon O, Seligsohn U. Prerequisites for recombinant factor VIIa-induced thrombin generation in plasmas deficient in factors VIII, IX or XI. J Thromb Haemost. 2006; 4:192-200. [PubMed: 16409469] 
23. Dargaud Y, Bordet JC, Francillon S, Negrier C. Haemophilia patients exhibit prolonged coagulation time but normal lag time of thrombin generation test: are these results really discordant? Thromb Haemost. 2007; 97:675-676. [PubMed: 17393033]

24. Wolberg AS. Thrombin generation and fibrin clot structure. Blood Rev. 2007; 21:131-142. [PubMed: 17208341]

25. He S, Ezban M, Bark N, Persson E, Hedner U. Fibrin gel structure obtained with a FVIIa analogue with enhanced FX-activating potential in haemophilia. Thromb Haemost. 2009; 102:790-792. [PubMed: 19806268]

26. Blomback B, Carlsson K, Hessel B, Liljeborg A, Procyk R, Aslund N. Native fibrin gel networks observed by 3D microscopy, permeation and turbidity. Biochim Biophys Acta. 1989; 997:96-110. [PubMed: 2752057]

27. Collet JP, Moen JL, Veklich YI, Gorkun OV, Lord ST, Montalescot G, et al. The alphaC domains of fibrinogen affect the structure of the fibrin clot, its physical properties, and its susceptibility to fibrinolysis. Blood. 2005; 106:3824-3830. [PubMed: 16091450]

28. Sixma JJ, van den Berg A. The haemostatic plug in haemophilia A: a morphological study of haemostatic plug formation in bleeding time skin wounds of patients with severe haemophilia A. Br J Haematol. 1984; 58:741-753. [PubMed: 6518139]

29. Weimer T, Wormsbacher W, Kronthaler U, Lang W, Liebing U, Schulte S. Prolonged in-vivo halflife of factor VIIa by fusion to albumin. Thromb Haemost. 2008; 99:659-667. [PubMed: 18392323]

30. Yatuv R, Dayan I, Carmel-Goren L, Robinson M, Aviv I, Goldenberg-Furmanov M, et al. Enhancement of factor VIIa haemostatic efficacy by formulation with PEGylated liposomes. Haemophilia. 2008; 14:476-483. [PubMed: 18393980]

31. Ghosh S, Sen P, Ezban M, Pendurthi UR, Rao LV. Activity and regulation of glycoPEGylated factor VIIa analogs. J Thromb Haemost. 2008; 5:336-346. [PubMed: 17092304]

32. Shah AM, Kisiel W, Foster DC, Nelsestuen GL. Manipulation of the membrane binding site of vitamin K-dependent proteins: enhanced biological function of human factor VII. Proc Natl Acad Sci U S A. 1998; 95:4229-4234. [PubMed: 9539719]

33. Persson E, Bak H, Ostergaard A, Olsen OH. Augmented intrinsic activity of Factor VIIa by replacement of residues 305, 314, 337 and 374: evidence of two unique mutational mechanisms of activity enhancement. Biochem J. 2004; 379:497-503. [PubMed: 14686879]

34. Sorensen B, Persson E, Ingerslev J. Factor VIIa analogue (V158D/E296V/M298Q-FVIIa) normalises clot formation in whole blood from patients with severe haemophilia A. Br J Haematol. 2007; 137:158-165. [PubMed: 17391496]

35. Brophy DF, Martin EJ, Nolte ME, Kuhn JG, Carr ME Jr. Effect of recombinant factor VIIa variant (NN1731) on platelet function, clot structure and force onset time in whole blood from healthy volunteers and haemophilia patients. Haemophilia. 2007; 13:533-541. [PubMed: 17880440]

36. Ghosh S, Ezban M, Persson E, Pendurthi U, Hedner U, Rao LV. Activity and regulation of factor VIIa analogs with increased potency at the endothelial cell surface. J Thromb Haemost. 2007; 5:336-346. [PubMed: 17092304]

37. Petersen LC, Karpf DM, Agerso H, Hermit MB, Pelzer H, Persson E, et al. Intravascular inhibition of factor VIIa and the analogue NN1731 by antithrombin. Br J Haematol. 2010

38. Viuff D, Ezban M, Lind V, Dillow A, Raymer R, Merricks E, et al. Effect of rFVIIa and NN1731 (RFVIIa analogue) on thromboelastography (TEG) in whole blood obtained from haemophilia A dogs. J Thromb Haemost. 2007; 5 P-W-113.

39. Hedner U. Dosing with recombinant factor VIIa based on current evidence. Semin Hematol. 2004; 1:35-39. [PubMed: 14872419]

40. Turecek PL, Varadi K, Keil B, Negrier C, Berntorp E, Astermark J, et al. Factor VIII inhibitorbypassing agents act by inducing thrombin generation and can be monitored by a thrombin generation assay. Pathophysiol Haemost Thromb. 2003; 33:16-22. [PubMed: 12853708]

41. Telgt DSC, Macik BG, McCord DM, Monroe DM, Roberts HR. Mechanism by which recombinant factor VIIa shortens the aPTT: Activation of factor X in the absence of tissue factor. Thromb Res. 1989; 56:603-609. [PubMed: 2626745] 
42. Hedner U. Factor VIIa and its potential therapeutic use in bleeding-associated pathologies. Thromb Haemost. 2008; 100:557-562. [PubMed: 18841276]

43. Livnat T, Martinowitz U, Zivelin A, Seligsohn U. Effects of factor VIII inhibitor bypassing activity (FEIBA), recombinant factor VIIa or both on thrombin generation in normal and haemophilia A plasma. Haemophilia. 2008; 14:782-786. [PubMed: 18371162]

44. Bevers EM, Comfurius P, Zwaal RF. Changes in membrane phospholipid distribution during platelet activation. Biochim Biophys Acta. 1983; 736:57-66. [PubMed: 6418205]

45. Campbell RA, Fischer TH, Wolberg AS. A novel approach to improving recombinant factor VIIa activity with a preserved platelet preparation. Br J Haematol. 2007; 138:82-93. [PubMed: 17555451] 


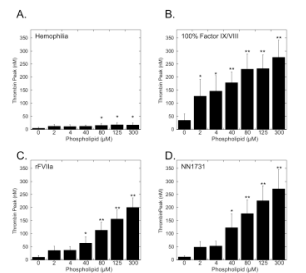

Figure 1. Like rFVIIa, NN1731-dependent thrombin generation depends on the phospholipid concentration

Thrombin generation was initiated by incubating re-calcified plasmas with $1 \mathrm{pM}$ TF and phospholipids at the concentrations indicated. Plasmas from eight different hemophilia A patients were tested, not all donors were tested at all conditions. Graphs show thrombin peaks $( \pm$ SD) in hemophilia PPP (A), or hemophilia A PPP supplemented with $100 \%$ factor VIII (B), $50 \mathrm{nM} \mathrm{rFVIIa} \mathrm{(C)} \mathrm{or} 5 \mathrm{nM}$ NN1731 (D), for 5-8 trials at each concentration. $* \mathrm{p}<0.05, * * \mathrm{p}<0.0001$ 


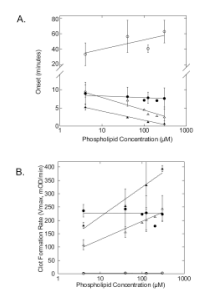

Figure 2. Both rFVIIa and NN1731 shorten the onset and increase rate of clot formation in a phospholipid-dependent manner

Clotting was initiated by incubating re-calcified hemophilic PPP with phospholipids and TFbearing monocytes, in the presence of 100\% factors VIII/IX, $50 \mathrm{nM}$ rFVIIa, $5 \mathrm{nM}$ NN1731, or buffer. Plasmas from six hemophilic patients were tested (five hemophilia A and one hemophilia B). Clot formation was monitored by turbidity at $405 \mathrm{~nm}$. A) Onset and B) Rate of clot formation $( \pm$ SEM). In experiments where samples did not clot, the onset was assigned to the final time point recorded (120 minutes). Symbols are: Hemophilia (open circles), $100 \%$ factors VIII/IX (closed circles), $50 \mathrm{nM} \mathrm{rFVIIa} \mathrm{(open} \mathrm{triangles),} 5 \mathrm{nM}$ NN1731 (closed triangles). 
A.

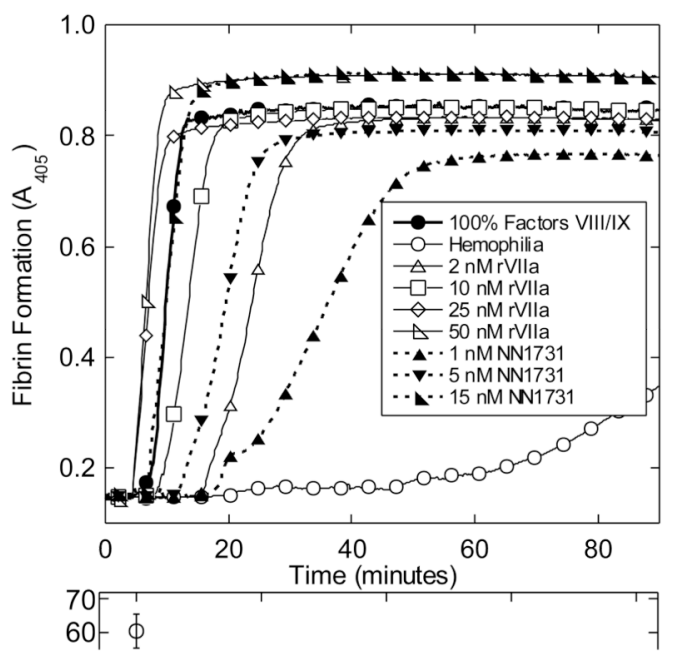

B.

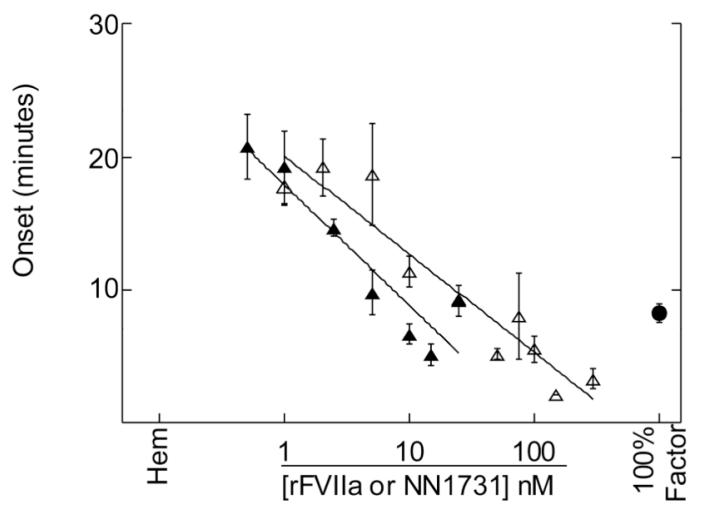

C.

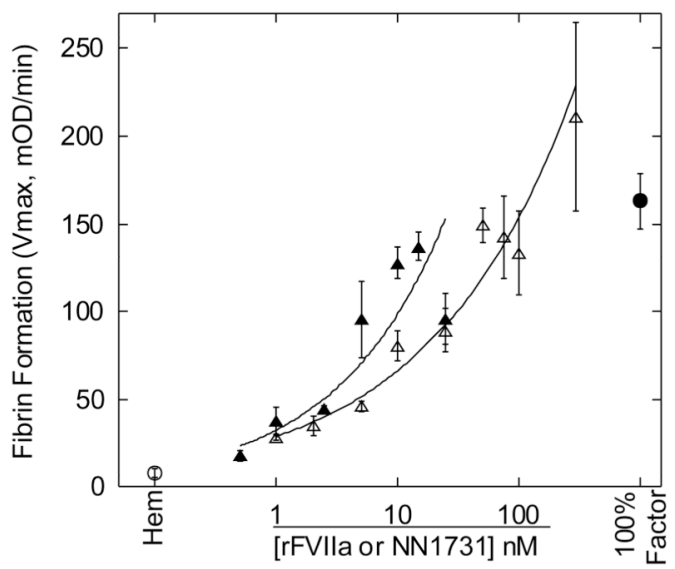

Figure 3. Compared to rFVIIa, lower concentrations of NN1731 shorten the onset and increase the rate of clot formation

Clotting was initiated by incubating re-calcified plasma with $125 \mu \mathrm{M}$ phospholipids and TFbearing monocytes and monitored by turbidity at $405 \mathrm{~nm}$. A) Clot formation from a single experiment, representative of separate experiments with six plasma donors (five hemophilia A, one hemophilia B). Symbols for panel A are indicated in the inset. B) Onset and C) Rate of clot formation from 1-23 clots at each condition $( \pm$ SEM). Symbols for B and C are: Hemophilia (open circle), 100\% factors VIII/IX (closed circle), rFVIIa (open triangles), NN1731 (closed triangles). 


\section{A.}
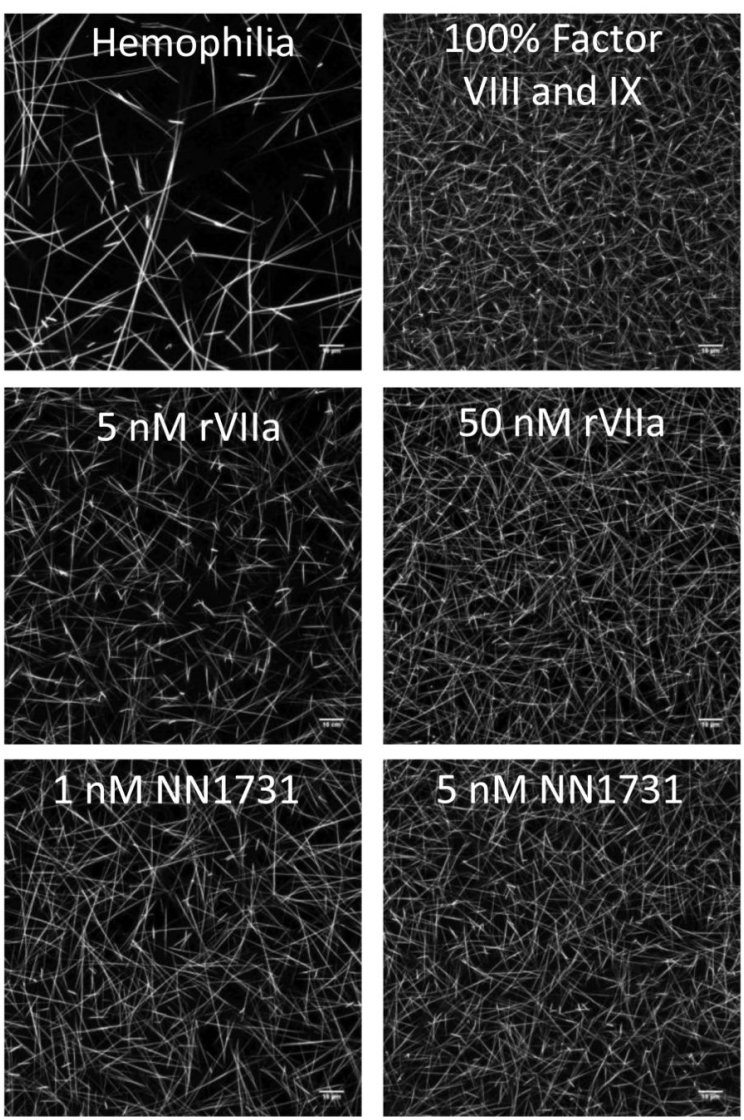

B.

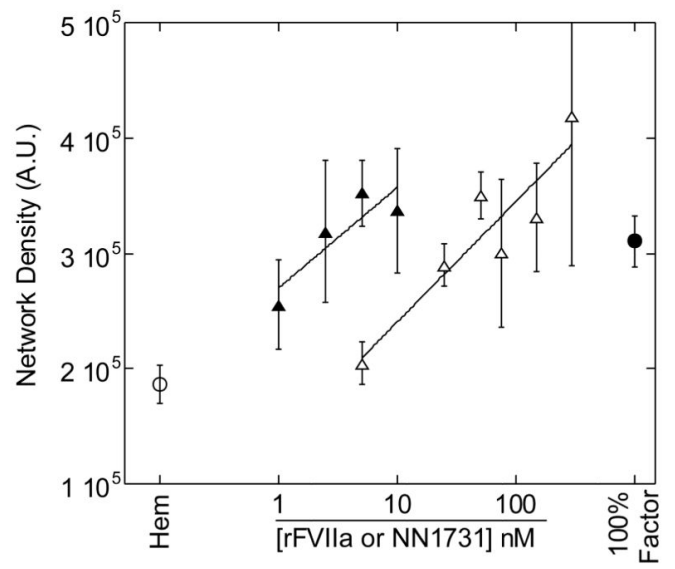

Figure 4. Compared to rFVIIa, lower concentrations of NN1731 increased fibrin network density Clotting was initiated by incubating re-calcified plasma with $125 \mu \mathrm{M}$ phospholipids and TFbearing monocytes in Labtek II glass chamber slides. Laser scanning confocal microscopy was performed as described in Methods. A) Confocal micrographs (z-projections of 30 individual slices) of clots formed in hemophilia PPP, or hemophilia PPP supplemented with $100 \%$ factors VIII/IX, rFVIIa, or NN1731. Images are from a single experiment, representative of experiments with six hemophilia patients (five hemophilia A, one hemophilia B). B) Fiber density (arbitrary units, A.U. \pm SEM) of 2-19 clots at each concentration. Symbols for panel B are: Hemophilia (open circle), 100\% factors VIII/IX (closed circle), rFVIIa (open triangles), NN1731 (closed triangles). 


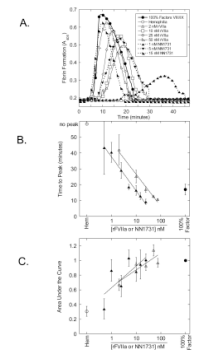

Figure 5. NN1731 improved resistance to fibrinolysis at lower concentrations than were required of rFVIIa

Clotting was initiated by incubating re-calcified hemophilic PPP with TF-bearing monocytes, $125 \mu \mathrm{M}$ phospholipids, and $1 \mu \mathrm{g} / \mathrm{mL}$ tPA. Clot formation (turbidity increase) and clot lysis (turbidity decrease) were monitored at $405 \mathrm{~nm}$. A) Fibrin formation and lysis from a single experiment, representative of experiments with six different plasma donors (five hemophilia A, one hemophilia B). Symbols for panel A are indicated in the inset. B) Time to peak of turbidity. In experiments where samples did not clot, the time to peak was assigned to the final time point recorded (60 minutes). C) Area under the curve ( \pm SEM) using 3-11 clots at each condition were normalized with " $100 \%$ factor" equal to 1 for each experiment. Symbols for B and C are: Hemophilia (open circle), 100\% factors VIII/IX (closed circle), rFVIIa (open triangles), NN1731 (closed triangles). 


\section{TABLE I}

Estimated Geometric Mean Concentrations Indistinguishable from 100\% Factors VIII and IX.

\begin{tabular}{|c|l|c|c|}
\hline \multicolumn{1}{|c|}{ Assay } & Parameter & nM rFVIIa (95\% CI) & nM NN1731 (95\% CI) \\
\hline \multirow{3}{*}{ Clot Formation and Structure } & Onset & $24.5(12.2-50.1)$ & $8.6(4.1-19.8)$ \\
\cline { 2 - 4 } & Formation Rate & $74.3(34.7-183.1)$ & $31.2(13.1-96.7)$ \\
\cline { 2 - 4 } & Fibrin Density & $34.5(3.1-172.5)$ & $2.9(0.32-22.3)$ \\
\hline \multirow{2}{*}{ Fibrinolysis } & Time to Peak Turbidity & $29.7(14.8-61.9)$ & $9.0(4.5-19.3)$ \\
\cline { 2 - 4 } & Area Under Curve & $37.1(5.0-467.1)$ & $11.3(1.7-182.5)$ \\
\hline
\end{tabular}


TABLE II

Relative Activity of NN1731 versus rFVIIa

\begin{tabular}{|c|l|l|}
\hline \multicolumn{1}{|c|}{ Assay } & Clot Parameter & Relative Increased Activity (95\% CI) \\
\hline \multirow{4}{*}{ Clot Formation and Structure } & Onset & $2.9(1.4-5.2)$ \\
\cline { 2 - 3 } & Formation Rate & $2.4(1.1-4.6)$ \\
\cline { 2 - 3 } & Fibrin Density & $12.1(1.0-76.9)$ \\
\hline \multirow{2}{*}{ Fibrinolysis } & Time to Peak Turbidity & $3.3(2.0-5.2)$ \\
\cline { 2 - 3 } & Area Under Curve & $3.3(0.6-12.7)$ \\
\hline
\end{tabular}

